\title{
EPIDEMIOLOGIA DO TRAUMA RAQUIMEDULAR EM EMERGÊNCIAS PÚBLICAS NO MUNICÍPIO DO RIO DE J ANEIRO
}

\author{
Spinal cord inj ury epidemiology in public emergency rooms in the municipality of Rio de \\ J aneiro \\ Epidemiología de trauma espinal en emergencias públicas del municipio de Rio de J aneiro
}

Talami Sayole Costa Santos ${ }^{1}$

Raphael Mendonça Guimarães²

Samyra Fábregas Boeira ${ }^{3}$

\section{RESUMO}

0 objetivo do presente estudo foi avaliar as taxas de mortalidade por trauma raquimedular (TRM) e estimar padrões diferenciados de características das internações hospitalares por TRM em hospitais públicos municipais e estaduais no município do Rio de Janeiro. Foram analisados dados do SIH-SUS sobre valor médio das internações, média de permanência, proporção de internações e taxa de mortalidade de hospitais municipais e estaduais, no período de 1996 a 2011 . Em relação ao valor gasto nas internações, média de permanência e número de internação, a rede estadual apresentou um maior comparativo em relação à rede municipal. Já em relação à mortalidade dos pacientes, os hospitais estaduais tiveram uma menor taxa de mor talidade. As evidências apontadas proporcionarão reflexões sobre a distribuição dos casos, taxa de mortalidade e o tipo de atendimento demandado, contribuindo para a organização da rede assistencial de emergências do município do Rio de Janeiro.

Palavras-chave: Trauma. Coluna vertebral. Serviços médicos de emergência. Epidemiologia descritiva. Enfermagem.

\begin{abstract}
The aim of this study was to assess mortality rates for spinal cord injury $(\mathrm{SCl})$ and to estimate the characteristics of different patterns of hospital admissions for TRM in municipal and state public hospitals in the city of Rio de Janeiro. We analyzed data from SIH-SUS on average of admissions, length of stay, hospitalization and mortality rates of municipal and state hospitals, from 1996 to 2011. Regarding the amount spent on hospital admissions, length of stay and number of admissions, the state system had a higher comparative regarding the municipal net. In relation to patient mortality, the state hospitals had a lower mortality rate. The evidence presented will provide reflections on the distribution of cases, mortality rate and the type of care required, contributing to the organization's emergency care network in the municipality of Rio de Janeiro.
\end{abstract}

Keywords: Trauma. Spine. Emergency Medical Services. Epidemiology, Descriptive. Nursing.

\section{Resumen}

El objetivo del presente estudio fue evaluar las tasas de mortalidad por trauma espinal (TRE) y estimar las características de los diferentes patrones de ingresos hospitalarios por TRE en hospitales públicos, municipales y estaduales, en la ciudad de Rio de Janeiro. Se analizaron los datos del SIH-SUS sobre el valor promedio de hospitalizaciones, la duración de la estancia media, la proporción de los ingresos hospitalarios y la mortalidad en los hospitales municipales y estaduales en el período de 1996 a 2011. En relación a la cantidad gastada en los ingresos hospitalarios, duración de la estancia y la hospitalización, la red estadual presentó un mayor comparativo en relación con la red municipal. En cuanto a la mortalidad de los pacientes, los hospitales del estado tuvieron una tasa de mortalidad más baja. Las evidencias apuntadas van a proporcionar reflexiones sobre la distribución de los casos, la tasa de mortalidad y el tipo de atención requerida, lo que contribuye a la organización del sistema de atención sanitaria de emergencia en el municipio de Rio de Janeiro.

Palabras clave: Trauma. Columna Vertebral. Servicios Médicos de Urgencia. Epidemiología descriptiva. Enfermería.

\footnotetext{
'Enfermeira. Aluna de Pós-Graduação em Promoção da Saúde e Desenvolvimento Social. ENSP - Escola Nacional De Saúde Pública Sergio Arouca. Rio de Janeiro-RJ. Brasil. E-mail: talamisayole@hotmail.com; ²Doutor em Saúde Coletiva. Professor Adjunto. Universidade Federal do Rio de Janeiro. Instituto de Estudos em Saúde Coletiva. Rio de Janeiro - RJ, Brasil. E-mail: raphael@iesc.ufrj.br; ${ }^{3}$ Acadêmica de Enfermagem. Universidade Federal do Rio de Janeiro. Escola de Enfermagem Anna Nery. Rio de Janeiro - RJ. Brasil. E-mail: sammyboeira@gmail.com
} 


\section{INTRODUÇÃO}

0 número de pessoas com lesões medulares vem aumentando nos últimos anos, e tal fato frequentemente é atribuído às causas externas, como a violência urbana e os acidentes de trânsito ${ }^{1}$ Os fatores que mais contribuem para 0 quantitativo crescente de lesados medulares são provenientes de lesões traumáticas, como ferimentos por armas de fogo, armas brancas, acidentes de trânsito, quedas e mergulho em águas rasas; o restante tem causa não traumática, como doenças degenerativas, tumores e malformações ${ }^{1}$. Já com relação ao perfil demográfico, o traumatismo raquimedular tem incidência maior no sexo masculino, na faixa etária dos adultos jovens, sem distinção de camada social2,3.

A medula espinal controla importantes funções do organismo; sendo assim, qualquer injúria medular leva a danos neurológicos ${ }^{4}$. Após a fase de choque medular, as funções medulares perdidas são irrestauráveis. 0 indivíduo precisa de avaliações, acompanhamento e cuidados específicos; por isso, faz-se necessário o acompanhamento de uma equipe multidisciplinar ${ }^{5}$. Tal equipe tem como função atuar no processo de reabilitação para minimizar ou atenuar as barreiras impostas pela lesão, aumentar a possibilidade de inclusão social, diminuir a dependência, estimular as aptidões preservadas e prevenção de agravos e possibilitar a reinserção social ${ }^{4}$.

A lesão medular é um evento incapacitante, que afeta não só o lesado medular, mas também sua família, já que engloba tudo que diz respeito à "perda da saúde" e a aquisição de limitações. Tudo isso envolve a mudança de hábitos e expectativa de vida, dependência, restrições, reeducação e a implementação no complexo processo de reabilitação $0^{5}$.

Os acidentes não são previsíveis, por isso devem ser tomadas medidas para prevenir tais episódios ${ }^{6}$; quando eles acontecem, as intervenções devem rápidas e objetivas para estabilizar e minimizar os danos. Independente de as lesões serem ortopédicas, cerebrais, neurológicas, medulares, faz-se necessário que o resgate seja feito por equipes especializadas, e não por transeuntes ou pessoas envolvidas no mesmo acidente, já que estas pessoas não sabem avaliar a complexidade. Diante de tal fato, é preciso que equipes especializadas prestem socorro para as emergências ${ }^{3}$.

0 atendimento do paciente deve ser iniciado logo no local da lesão, com a avaliação clínica, por meio do reconhecimento das lesões do paciente e prevenção de lesões associadas durante o resgate e transporte até o local do atendimento definitivo. A avaliação do paciente compreende a história, exame físico, exame neurológico, exame radiológico e outros exames complementares quando necessários ${ }^{7}$.

A incidência de lesão medular traumática no Brasil é desconhecida, pois esta condição não é sujeita à notificação e há poucos dados e trabalhos publicados a respeito da epidemiologia da lesão medular ${ }^{5}$. Entretanto, há um conhecimento, ainda que incipiente, sobre o tema, utilizando os dados referentes às internações hospitalares que têm o traumatismo raquimedular como causa.

Os pronto-socorros ainda são importante porta de entrada para assistência médica, e boa parte da população usuária do SUS busca assistência por meio de consultas de pronto-atendimento nos pronto-socorros dos hospitais, em vez de buscar esse tipo de atendimento na rede básica de saúde (porta de entrada presumida). É fato que o padrão de utilização de serviços de saúde de um determinado grupo populacional é principalmente explicado por seu perfil de necessidade em saúde, porém as preferências e escolhas dos usuários influenciam na utilização dos serviços tanto quanto a forma como a rede está estruturada. Essa constatação precisa ser incorporada por gestores e profissionais para diminuir a expectativa de que a resolução estrutural da rede de serviços de saúde seja condição suficiente para corrigir o inadequado padrão de sua utilização8.

Diante disso, o objetivo do estudo é avaliar as taxas de mortalidade por trauma raquimedular (TRM) e estimar padrões diferenciados de características das internações hospitalares por TRM em hospitais públicos municipais e estaduais no município do Rio de Janeiro entre 1996 e 2011.

\section{METODOLOGIA}

Este é um estudo epidemiológico que utilizou dados secundários provenientes do Sistema de Internações Hospitalares (SIH) para a ocorrência de internações por trauma raquimedular (TRM) no Município do Rio de Janeiro entre 1996 e 2011, de acordo com a natureza jurídica da emergência pública em questão (municipal ou estadual).

Os dados disponíveis são oriundos do Sistema de Informações Hospitalares do SUS-SIH/SUS, gerido pelo Ministério da Saúde, através daSecretaria de Assistência àSaúde, em conjunto com as Secretarias Estaduais de Saúde e as Secretarias Municipais de Saúde, sendo processado pelo DATASUS - Departamento de Informática do SUS, da Secretaria Executiva do Ministério da Saúde. Foram utilizados dados sobre o número de internações, valor médio das Autorizações de Internação Hospitalar (AlH) pagas e média dos dias de permanência internados, bem como a mortalidade por TRM, no período de 1996 a 2011.

A população do estudo corresponde às pessoas que foram internadas em emergência pública no município do Rio de Janeiro, cuja internação foi codificada pela APAC (Autorização para Procedimento de Alta Complexidade) ou pela AlH (Autorização para Internação Hospitalar) como: tratamento clínico para trauma raquimedular.

Utilizou-se o pacote estatístico Stata 11.0 para a descrição e análise de dados.

\section{RESULTADOS}

A Tabela 1 descreve os valores médios dos indicadores do SIH-SUS para Internação por Trauma Raquimedular segundo natureza jurídica no Rio de Janeiro entre 1996 e 2011. 
Tabela 1: Valores Médios dos indicadores do SIH-SUS para Internação por Trauma Raquimedular segundo natureza jurídica. Rio de Janeiro, 1996 - 2011

\begin{tabular}{llllll}
\hline Indicadores & Natureza Jurídica & Média & DP & IC $95 \%$ & p valor* \\
\hline Valor médio de AlH & Estadual & 653,79 & 253,27 & $518,83-788,75$ & $0,009^{*}$ \\
(em reais) & Municipal & 619,86 & 143,11 & $543,60-696,12$ & \\
Média de permanência (em dias) & Estadual & 38,77 & 12,32 & $32,20-45,33$ & $<0,001^{*}$ \\
& Municipal & 19,15 & 8,03 & $14,88-23,44$ & \\
Proporção de internações (em \%) & Estadual & 50,77 & 22,22 & $38,93-62,62$ & $0,018^{* *}$ \\
& Municipal & 41,28 & 20,39 & $30,14-52,14$ & \\
Taxa de mortalidade & Estadual & 13,32 & 8,97 & $8,54-18,11$ & $0,002^{* *}$ \\
(a cada 1.000 habitantes) & Municipal & 18,75 & 11,45 & $12,65-24,86$ & \\
\hline
\end{tabular}

* Obtido por meio de teste de ANOVA, calculando-se a diferença ano a ano.

** Obtido por meio de teste de Qui-quadrado de Pearson, calculando-se a diferença ano a ano.

Observa-se que, em média, as internações em hospitais estaduais geram um maior tempo de internação e, por conta disso, um maior custo por internação. Proporcionalmente, há mais internações para tratamento clínico de lesões medulares em emergências estaduais do que municipais. Entretanto, a taxa de mortalidade tendo como causa primária o TRM é maior em hospitais municipais.

0 Gráfico 1 mostra o valor médio de AlH para tratamento de TRM por ano competência e natureza no município do Rio de Janeiro entre 1996 e 2011.

Gráfico 1: Valor médio de AlH para tratamento de TRM por ano competência e natureza. Município do Rio de Janeiro, $1996-2011$.

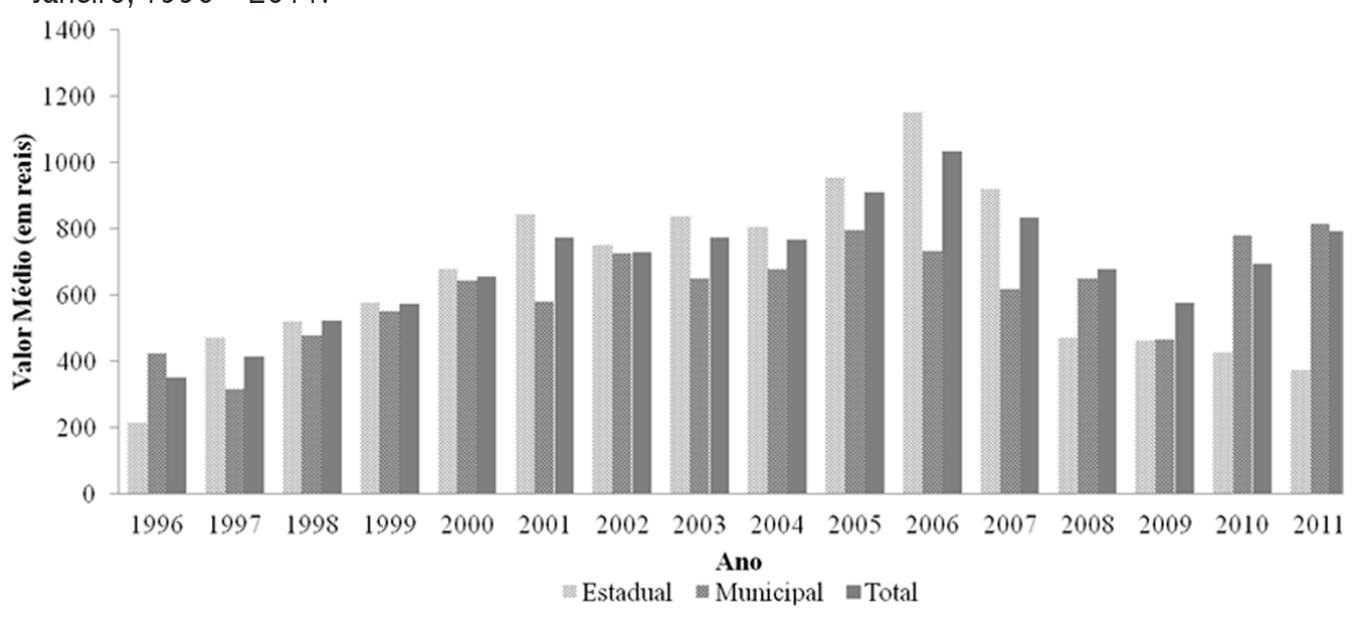

Observa-se que o valor gasto com a internação na rede estadual é superior ao da municipal. Entretanto, tal diferença mostrou-se oscilante ao longo do período analisado e é estatisticamente significativa $(p=0,009$, Tabela 1$)$. A partir de 2008, entretanto, esta situação mudou, e os hospitais municipais

passaram a modificar seu perfil, gerando maiores valores pagos por AlH, comparados aos hospitais estaduais.

0 Gráfico 2 apresenta a média de permanência de internação para tratamento de TRM por ano competência e natureza jurídica no município do Rio de Janeiro entre 1996 e 2011.

Gráfico 2: Média de permanência de internação para tratamento de TRM por ano competência e natureza jurídica. Município do Rio de Janeiro1996 - 2011

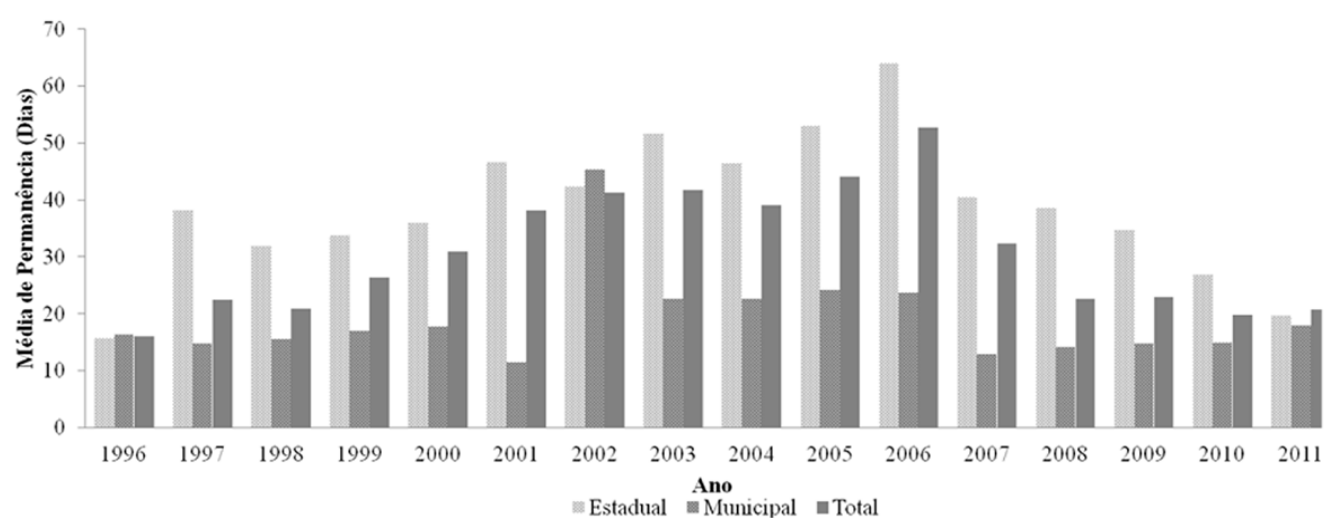


Durante todo o período, o tempo médio de permanência foi maior nos hospitais estaduais, com exceção no ano de 2002. A média de internação nos hospitais estaduais é de pouco mais de um mês, e na rede municipal, de menos de um mês. Cabe ressaltar que, a partir de 2008, para ambas as naturezas jurídicas (estadual e municipal), 0 tempo médio de permanência diminuiu, resultado da qualificação do atendimento pré-hospitalar.

Já o Gráfico 3 mostra as Internações Hospitalares para tratamento de TRM por ano de competência e natureza jurídica no município do Rio de Janeiro entre 1996 e 2011.

Gráfico 3: Internaç̃̃es hospitalares para tratamento de TRM por ano de competência e natureza jurídica. Município do Rio de Janeiro, 1996 - 2011

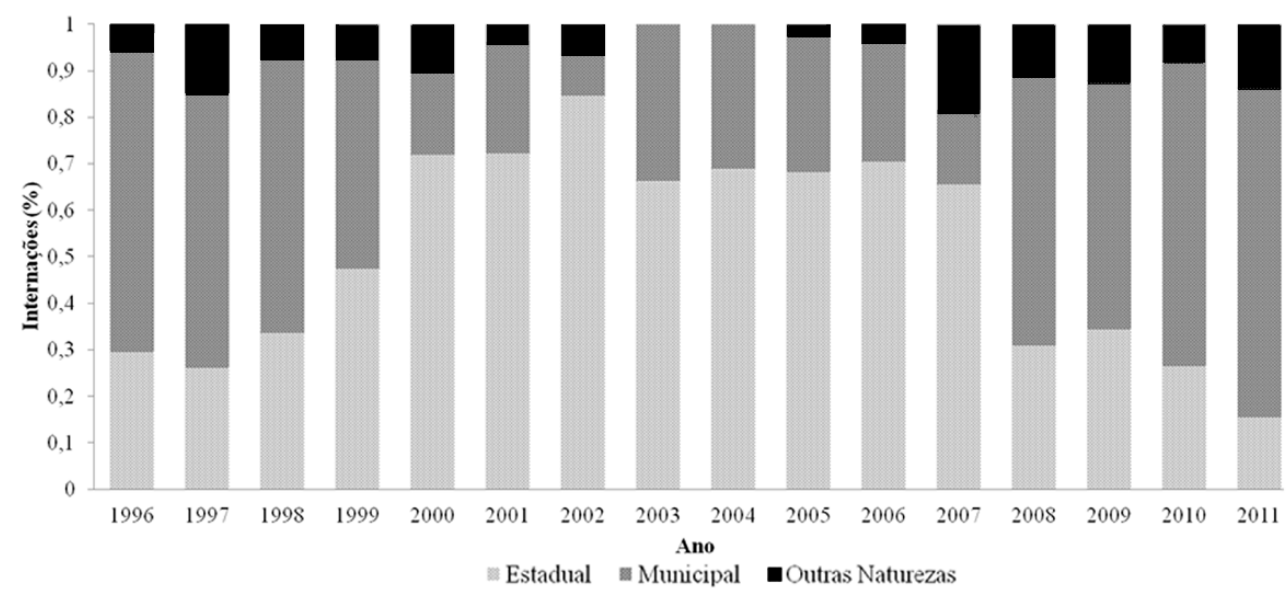

A maioria das internações hospitalares tendo como causa o TRM ocorreu em hospitais estaduais. Entretanto, bem como as demais características avaliadas, gradativamente esta diferença tem diminuído, especialmente a partir de 2008.
Finalmente, o Gráfico 4 evidencia Taxa mortalidade por TRM segundo ano competência e natureza jurídica no Município do Rio de Janeiro entre 1996 e 2011.

Gráfico 4: Taxa mortalidade por TRM segundo ano competência e natureza jurídica. Município do Rio de Janeiro, 1996 - 2011

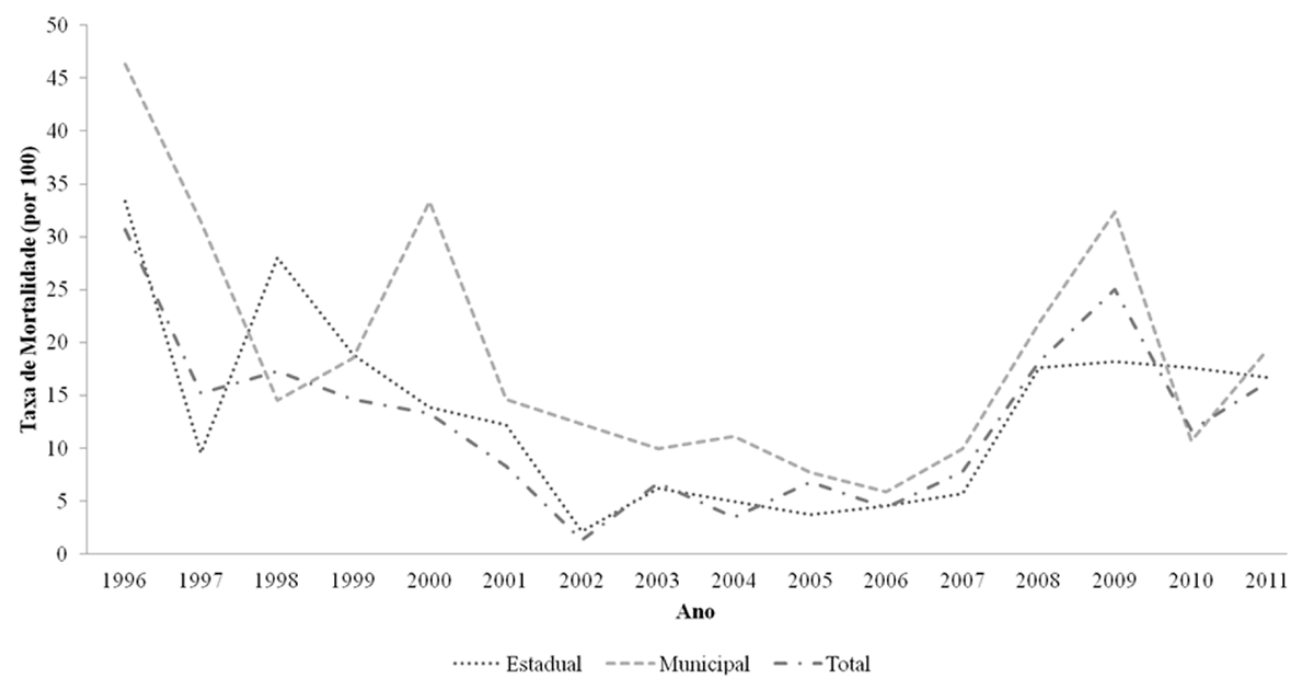

Observa-se que, durante o período analisado, houve uma diminuição crescente do número de óbitos no período de 1996 a 2002. No período entre 2002 e 2011, os dados mostram oscilações em relação aos valores da taxa de mortalidade, com queda importante entre 2002 e 2007, e novo crescimento entre 2007 e 2011.
Observa-se, portanto, que 2007-2008 foi um biênio que apresenta um efeito de período, modificando o padrão de ocorrência dos indicadores para as internações para tratamento de TRM. 


\section{DISCUSSÃO}

Os dados apresentados descrevem diferenças no perfil das internações por trauma raquimedular no município do Rio de Janeiro. Tais indicadores podem ser influenciados por fatores socioeconômicos, epidemiológicos e demográficos que determinam o perfil da demanda hospitalar ao SUS, além de políticas públicas de atenção à saúde ${ }^{9}$.

De acordo com o Gráfico 1, nota-se que o valor gasto com a internação na rede estadual é superior ao da municipal. Avalia-se que o valor gasto com internação é diretamente ligado ao tempo de internação e à complexidade do tratamento.

Cada internação hospitalar realizada no SUS em 2000 custou, em média, $R \$ 409$, com variações de $R \$ 281$, na região Norte, a $\mathrm{R} \$ 484$, na região Sudeste. Em 2005, o menor gasto médio ocorreu em cuidados prolongados, na região Norte (R\$ 103), e o maior também em cuidados prolongados, na região Sudeste ( $\mathrm{R} \$ 4.546)$. Entre todas as especialidades, o valor médio varia de $R \$ 418$, na região Norte, a $R \$ 706$, na região Sudeste. Entre 2000 e 2005, há um aumento de $49 \%$ no valor médio, variando de $-3 \%$ na reabilitação e $108 \%$ em cuidados prolongados. Nas especialidades básicas (clínica médica, pediatria, obstetrícia e clínica cirúrgica), o aumento do valor médio é de cerca de 50\%, com exceção da clínica médica, 37\%. Pode ser observado ainda o menor valor médio da psiquiatriahospital dia em relação à psiquiatria tradicional ${ }^{11 .}$.

Conforme pode ser observado no Gráfico 2, o tempo médio de permanência foi maior nos hospitais estaduais, com exceção no ano de 2002. A média de internação nos hospitais estaduais gira é de pouco mais de um mês, e na rede municipal, de menos de um mês, tal diferença é estatisticamente significativa $(\mathrm{p}<0,001)$. Após análise da figura, tais dados levam à reflexão dos motivos para esse fato, como a diferença entre 0 tratamento na rede municipal e estadual; a relação entre 0 tempo de permanência e o número de óbitos; o tempo de sobrevida; e o nível de gravidade dos pacientes.

Conjectura-se que a clientela com lesão medular que ingressa no serviço público através das emergências apresente um grau de complexidade significativo, com risco de morte e grande necessidade de cuidados complexos; isso pode ser evidenciado até mesmo pela figura relacionada aos custos. Diante dessas características de tal clientela, pode-se pensar que o tempo de permanência está diretamente ligado ao tempo de sobrevida e à complexidade de agravos de determinado paciente.

Imagina-se que o tempo de permanência é diretamente proporcional aos custos. Uma vez que um paciente permaneça mais tempo no hospital, isto encarece o custo de internação. Os procedimentos especiais também se associam à variação do custo total de internação. Instrumentos mais sofisticados implicam na elevação natural do custo de internação. Isso não significa dizer, obviamente, que não se recomende a adoção de instrumental mais sofisticado na rede hospitalar. 0 uso de leitos de UTI e os casos que requerem cirurgia significam um aumento considerável dos gastos, o que diz respeito ao requerimento de aparelhos mais complexos e de pessoal técnico habilitado para seu uso. É claro que estes casos se referem a acidentes mais graves, muitas vezes com sequelas para o resto da vida, ou até, resultando em morte. Estes procedimentos, então, estariam ligados à variação da gravidade das lesões ${ }^{12,13}$.

É necessário dizer que o controle dos custos não se dá somente no nível de administração hospitalar, mas também no nível mais amplo da administração pública, como campanhas de reeducação para 0 trânsito, formas mais rigorosas na liberação de carteiras de habilitação, no controle de porte e aquisição de armas de fogo ${ }^{12}$.

Após análise do período de 1996 a 2011, a maioria das internações hospitalares tendo como causa o TRM ocorreu em hospitais estaduais, em torno de $50 \%$ das internações durante o intervalo de tempo analisado. Tal fato evidencia que o quantitativo é muito maior que os hospitais das demais naturezas; trata-se de uma diferença estatisticamente significativa $(p=0,018)$. Em seguida vem os hospitais municipais que se apresentam na maioria dos anos em segundo lugar. Entretanto, paulatinamente esta diferença tem diminuído, especialmente a partir de 2008.

Acredita-se que o número de internações esteja diretamente relacionado com a complexidade das internações, visto que, quanto maior a complexidade, maior o suporte necessário para manutenção do tratamento e cuidado da clientela. Fazendo um breve comparativo, uma unidade que acolha pacientes com maiores necessidades recebe um número menor de pacientes, já que demanda um número superior de cuidados do que uma unidade mais basal que consequentemente poderá absorver um quantitaivo maior de pacientes. Ainda, refere-se à melhoria no atendimento às emergências e urgências no município, dado o incentivo de gestão e o aumento da cobertura das Unidades de ProntoAtendimento (UPAs), determinando uma mudança na rotina do fluxo para urgência e emergência na rede SUS.

Segundo Wennberg ${ }^{14}$, há estudos têm apontado a existência diferença nas taxas de utilização de serviços públicos de saúde entre regiões e prestadores, que não seriam explicadas por diferenças no perfil dos pacientes ou da necessidade de saúde, mas estariam relacionadas à organização da oferta e das preferências por determinadas práticas médicas ${ }^{14}$. Por exemplo, em razão de complicações secundárias que os indivíduos com lesão medular podem apresentar, há maior risco de necessitarem de nova hospitalização ao longo da vida, quando comparados com a população que não é portadora de lesão medular ${ }^{15}$. A perda do controle esfincteriano (vesical e intestinal), a alteração da sensibilidade com a formação de úlceras de pressão, processos degenerativos osteoarticulares e fenômenos tromboembólicos, são as maiores causas de morbimortalidade em indivíduos com lesão medular ${ }^{16}$.

Finalmente, durante o período analisado, houve uma diminuição crescente do número de óbitos no período de 1996 
a 2002. No período entre 2002 e 2011 , os dados mostram oscilações em relação aos valores da taxa de mortalidade, com queda importante entre 2002 e 2007, e novo crescimento entre 2007 e 2011.

0 perfil de mortalidade do município do Rio de Janeiro, como no Brasil, vem mudando nas últimas décadas, ocorre o crescimento significativo da participação das causas externas. No Rio de Janeiro, a violência é, sobretudo, metropolitana, e contribui para esta situação o fato de a cidade ter sofrido, nas últimas décadas, os efeitos de uma urbanização desordenada, efeito de uma política urbana quase inexistente, destinando, aos migrantes recém-chegados e descendentes, moradias irregulares e sem infraestrutura ${ }^{10}$.

Em estudo sobre a mortalidade de pacientes com fratura da coluna cervical durante o período de hospitalização foram tiradas importantes conclusões acerca de fatores determinantes. A lesão neurológica grave do tipo paraplegia ou tetraplegia foi o principal fator de risco para a mortalidade dos pacientes com fratura da coluna cervical. A fratura-luxação da coluna cervical foi o principal tipo de fratura presente nos pacientes que morreram durante a hospitalização, principalmente entre a quinta e a sexta vértebras cervicais. $\mathrm{A}$ infecção respiratória foi a complicação mais frequente observada nos pacientes que morreram com fratura cervical durante a internação hospitalar ${ }^{17}$.

A partir de dezembro de 1997, as unidades hospitalares participantes do SUS (públicas, universitárias ou filantrópicas/privadas credenciadas), no Estado do Rio de Janeiro, enviam as informações das internações efetuadas através da AlH - Autorização de Internação Hospitalar, para os gestores municipais que, as integralizam, validam e compatibilizam com os tetos financeiros hospitalares. Posteriormente, estas são enviadas à SES-RJ para análise e geração do banco de dados estadual a ser encaminhado ao DATASUS, que gera os créditos referentes aos serviços prestados, formando uma valiosa Base de Dados contendo dados de grande parte das internações hospitalares realizadas no Brasilis.

0 presente estudo conta com algumas limitações: trabalhou-se com dados secundários, e isto impede que análises multivariadas mais rebuscadas sejam realizadas, pela ausência de informação das covariadas que potencialmente são variáveis confundidoras. Além disso, a qualidade da informação pode variar segundo nível de gestão, no tocante ao preenchimento das AlH e à precisão das informações dos prontuários para classificar o tipo de internação e o tempo médio de permanência. Entretanto, considerando o efeito ecológico das informações (dados a respeito da organização do sistema de saúde), a análise realizada parece apropriada ao tipo de inferência que se pretendia obter.

Deve-se mencionar que os dados utilizados provêm de um instrumento (AlHs) que é basicamente utilizado para o faturamento e não reflete a preocupação com outras questões.
Isso pode ser um fator influenciador dos resultados. As AlHs, apesar de suas falhas, podem ser um instrumento de ajuda para uma melhor administração dos recursos (humanos, financeiros e de material) dos hospitais públicos e conveniados. No que tange aos recursos humanos, por exemplo, algumas explicações para a variação no custo de internação residem nos seguintes aspectos: as equipes médicas podem ter composição diferenciada, incluindo ou não certos especialistas nos plantões; os pacientes podem ter atendimento de um número menor ou maior de especialistas, variando os custos. Outra implicação dos recursos humanos é que melhores treinamento e especialização de pessoal podem implicar, a médio e longo prazos, uma redução do custo total, em função de uma melhor utilização do material e dos instrumentos, bem como de maior eficácia nos processos clínicos de diagnose ${ }^{12}$.

\section{CONCLUSÃO}

0 aumento da demanda exige dos serviços de emergência uma (re)organização das equipes a fim de garantir um atendimento com maior complexidade. Torna-se necessário criar uma dinâmica de referência para serviços e instituições a fim de dar continuidade ao atendimento às vítimas que sofreram sequelas e que precisaram de apoio para a reabilitação. A emergência, por suas características, acaba fazendo o papel de reguladora do sistema, ou seja, depósito dos problemas não resolvidos.

A deficiência de dados, entretanto, constitui um obstáculo importante para o desenvolvimento de programas de segurança no trânsito e prejudica a configuração e análise do problema. Neste sentido, este estudo teve a intenção de realizar um diagnóstico situacional quanto ao perfil das internações hospitalares por TRM no Município do Rio de

\section{REFERÊNCIAS}

1.Venturini DA, Decésaro MN, Marcon SS. Alterações e expectativas vivenciadas pelos indivíduos com lesão raquimedular e suas famílias, São Paulo. Rev Esc Enferm USP. 2007; 41(4): 589-96.

2.Figueiró RFS. 0 paraplégico no mercado de trabalho: a percepção dos trabalhadores sem deficiência motora: reflexões para a enfermagem. Rio de Janeiro. UFRJ/ EEAN; 2007.

3.Acidentes de trânsito. Veículos: caracterização dos pacientes. [citado 2009 maio 27] Disponível em: http: //www.sarah.br .

4.Brunil DS, Strazzieri KC, Gumieiro MN, Giovanazzi R, Sá VG, Faro, ACM. Aspectos fisiológicos e assistenciais de enfermagem da reabilitação da pessoa com lesão medular, São Paulo. Rev Esc Enferm USP. 2004; 38(1): 71-9. 
5.Campos MF, Ribeiro AT, Listik S, Pereira CA, Sobrinho JA, Rapoport A. Epidemiologia do traumatismo da coluna vertebral no serviço de neurocirurgia do hospital Heliópolis. Rev Col Bras Cir. [on-line]. 2008; 35(2). [citado 2009 jun 10] Disponível em: http://www.scielo.br/rcbc.

6.Pereira MEMSM, Araujo TCCF.Estratégias de enfrentamento na reabilitação do traumatismo raquimedular. Arq Neuro-Psiquiatr. 2005; 63(2-B): 50207.

7.Soares B, Castro EMC, Reis PC. Análise dos resultados da reabilitação funcional no traumatismo raquimedular total de nível neurológico $C 5$ através da Medida de Independência Funcional - MIF: um estudo de caso; 2008. [citado 2009 jun 01] Disponível em: http://www.wgate.com.br/ conteudo/ medicinaesaude/fisioterapia/neuro/raquimedular_eduardo.htm.

8.0'Dwyer G, Matta IEA, Pepe VLE. Avaliação dos serviços hospitalares de emergência do estado do Rio de Janeiro. Rio de Janeiro: DAPS/ ENSP / FIOCRUZ; 2007.

9.Ministério da Saúde (BR). Sistema de Informações Hospitalares do SUS (SIH/SUS). Procedimentos hospitalares. [citado 2009 jun 28] Disponível em: http://www.saude.rj.gov.br/cgi/AihOrig2.htm.

10.Gomes LP, Melo ECP. Distribuição da mortalidade por acidentes de trânsito no município do Rio de Janeiro. Esc Anna Nery. 2007 jun; 11 (2): $289-95$.

11.Pinheiro RS, Travassos C, Gamerman D. Desigualdade no tratamento à fratura proximal de fêmur no Rio de Janeiro. Rev Bras Epidemiol. 2006; 9(3): 374-83.

12.Feijó MCC, Portela MC. Variação no custo de internações hospitalares por lesão: os casos dos traumatismos cranianos e acidentes por armas de fogo. Cad Saude Publica. 2001; 17(3): 627-37.

13. Ministério da Saúde (BR). Sistema de Informações Hospitalares do SUS-SIH/SUS). [citado 2009 abr 12] Disponível em http:// w3.datasus.gov.br/datasus/datasus.php.

14.Wennberg JE. On patient need, equity, supplier-induced demand, and the need to assess the outcome of common medical practices. Med Care, 1985; 23(5): 512-20.

15.Cardenas DD, Hoffman JM, Kirshblum S, McKinley W. Etiology and incidence of rehospitalization after traumatic spinalcord injury: a multicenter analysis. Arch Phys Med Rehabil. 2004; 85(11): 1757-763.

16.Meyer F, Vialle LR, Vialle EN, Bleggi-Torres LF, Rasera E, Leonel Alterações vesicais na lesão medular experimental em ratos. Acta Cir Bras. 2003; 18(3): 203-08.

17.0liveira AR, Avanzi 0. Estudo sobre a mortalidade de pacientes com fratura da coluna cervical durante o período de hospitalização. Rev Bras Ortop. 2002; 37(3): 89-96.

18.Cabral não investe o que foi previsto para a área da saúde. JB Online, 2007. [citado 2009 jun 20] Disponível em: http://www4.ensp.fiocruz.br/ informe/anexos/JB\%20-\%20segunda-feira.pdf 